\title{
Comparative Dynamics: Healthy Collectivities and the Pattern Which Connects
}

\author{
DARREN STANLEY \\ University of Windsor (Canada)
}

\begin{abstract}
In this paper, I introduce the notion of "comparative dynamics" and the importance of connectivity as an essential and vital underlying principle for healthy collectivities. Such a notion resonates with Gregory Bateson's idea of the "pattern which connects," suggesting not only the functional importance of connectivity as an aspect of a healthy organization at some given scale, but also connectivity as an important principle, which is the basis for how all living patterns are connected together. This paper ends with some reflections on why and how teachers experience stress and burnout as an absence of connectivity while highlighting its importance in the well-being of teachers in healthy learning organizations.
\end{abstract}

The pattern which connects is a metapattern. It is a pattern of patterns. It is that metapattern which defines the vast generalization that, indeed, it is patterns which connect. (Bateson, 1979)

\section{Introduction}

The historiography of dynamical systems, as Aubin and Dalmedico (2002) suggest, has shown that a "great surge of interest in dynamical systems theory" has emerged over time, especially since the 1970s, and is stretching well beyond the usually-taken-for-granted boundaries of mathematics, moving into other areas of study, as well as the popular press. To be sure, 
however, dynamical phenomena, broadly speaking, have been studied for centuries, but the late $20^{\text {th }}$ century has shown itself to be a rather important time for many scholars and researchers across various disciplines, including, of course, mathematics, but also the natural and social sciences, and the arts. In the past three decades, researchers in geology, biology, ecology, psychology, sociology, economics and organizational theory, to name a few disciplines, slowly have grown more attentive to the complex and apparently disorderly nature of what have since become known as complex systems or non-linear dynamical systems.

Of the many different conceptualizations of dynamical phenomena that have emerged during the $20^{\text {th }}$ century-for instance, general systems theory, dissipative systems theory, chaos theory, and self-organized criticality-the term "complex adaptive systems" (CAS) currently stands as one of the better known terms under the umbrella of non-linear dynamical systems. At the heart of CAS is the idea that large numbers of agents interacting locally give rise to their own structures, self-organizing in such a fashion so as to bring forth the possibility of larger dynamically coherent, persistent patterns. That is, in the absence of an overall "blueprint", globally emergent patterns can arise through local interactions for the on-going movement and unfolding of the system itself.

Studies of dynamical phenomena suggest that the concept of connectivity is an important aspect of, and for, complex phenomena in terms of coherence and communication, for instance. To begin, I invoke the notion of "comparative dynamics" and explore how the concept of connectivity plays an important part in the "health" of complex organizational collectivities.

\section{Comparative Dynamics}

Following other branches of comparative inquiry like "comparative anatomy," "comparative literature," "comparative education," and so on, the notion of "comparative dynamics" comes to mind as a way to help frame an approach to compare and understand the dynamics and dynamical patterns of a variety of different phenomena. As the word suggests, a "comparison" involves a likening of things where certain characteristics are highlighted for their similarities or differences between those things, with the aim of showing certain relative qualities. In addition, the other term, "dynamics," is concerned with the dynamical forces that make something happen. As a branch of physics, dynamics addresses the relation between the forces of a system and the ways in which the patterns of the system change temporally and spatially.

The focus of a comparative dynamics approach is, thus, on the similarities and differences of dynamical patterns that arise from within particular, 
and across various, scales of organizations of dynamical patterns (Stanley, $2004 ;$ 2005). For instance, the gait of a healthy human being and that of a person living with Huntington's disease (Hausdorff et al., 1997) and the dynamics of the heart, especially congestive heart failure and atrial fibrillation (Goldberger et al., 2002), are illustrations of patterns that are manifestations of both "complicated" and "complex" patterns. Although the kind of phenomena of interest here are not necessarily restricted to "complex" phenomena, what ought to be kept in mind is the need to compare patterns from the same kinds of phenomena. That is, the comparison ought to be between complex systems, or simple systems with simple systems, and so on. But more importantly, what ought to be remembered is that complex phenomena do bring forth a wide variety of different patterns, including seemingly simple, predictable and regular patterns. This, I wish to claim, is a reflection of the nature and kind of connectivity present.

As an analogy, the research in the area of Boolean networks by Stuart Kauffman (1995) illustrates the same idea. Whether by way of buttons tied with strings or light bulbs connected in a wired network, Kauffman has observed how particular network patterns create specific "portraits" of Boolean nets. More specifically, on one hand, sparsely connected networks manifest highly ordered patterns and, on the other hand, highly connected networks tend toward chaos. "Fine tuning" the network, however, allows the system to enter into a "phase transition regime" (Kauffman, 1995, p. 80) between ordered and chaos. In other words, the system has the capacity to move into a region that lies near the "edge of chaos."

Often when references to complex systems are made, comparisons and analogies across different kinds and scales of organization are invoked, comparing, for instance, bird flocks with termite colonies, human riots with bee swarms, and traffic jams with the growth of cancer cells. And so, for this reason, the term "comparative dynamics" has emerged. Comparisons that others have invoked to understand the nature of complex systems also have prompted medical researchers to think about healthy physiological systems in terms of CAS. Although the terms "health" and "healthy" may be subjective, there appears to be a connection between different physiological structures in terms of diverse dynamical tendencies (Kelso \& Engstrøm, 2006) for states of "health" and the presence of particular dynamical patterns for those states.

The introduction of the term "comparative dynamics" has emerged also from a realization that, under certain conditions, the dynamics of a particular complex phenomenon might give rise to tendencies for particular kinds of patterns which can be described as "healthy" or "unhealthy." The notion of an organizational dynamic described as "healthy" or "unhealthy" certainly can be illustrated through examples from the field of human physiology-for 
example, the physiological organization of the human heart and the human gait. Moreover, terms related to notions of health are, in fact, already used in more popular parlance to describe particular human relationships as with the notions of divorce, sick ideas and toxic workplace environments (Frost, 2003). Through the notion of comparative dynamics, therefore, the concept of dynamical organizational health can be extended to other scales of complex organization, which include the biological body, as well as other socially-, culturally-, politically-, and ecologically-organized bodies. But, even more, such organizations must not only be connected for purposes of coherence, for instance, but must be connected to one another to sustain life.

Connectivity, therefore, could be said to be a matter of and for vitality. In other words, in matters of health and disease (or illness), connectivity is not merely crucial —in one sense of the word "vital" - but is important to the overall picture of health of all living beings, as the etymology of "vitality" ought to remind us. Connectivity, therefore, "speaks" - albeit quietly when living beings are healthy and quite "loud and clear" in times of distress, illness and disease-in particular ways that reflect the nature of health for all living beings.

\section{Why Connectivity?}

Our own collusion with the world—out of sheer necessity—brings human beings into a complex set of connections, both with themselves through self-reflexivity and the world. As such, through one's perceptions of the world, human beings are already complicit in the creation of some sense of complexity-at-work. This is reflected, for example, in the various and diverse perspectives and ideas that human beings bring to one another in conversation. In a manner of speaking, therefore, it also may be through one's "mindset" of the world that one becomes disconnected from the world through particular conceptualizations of that world. Thus, a different mindset that draws one's attentions to the presence of various kinds of connections might give one cause to be suspicious of any kind of cutting up of the world that one might do.

As Ralph Stacey (2003) argues, human beings cannot self-regulate in isolation from the rest of the world: human beings require other human beings to come into contact with one another and to form relationships. In other words, connections are a matter of survival for human beings. Such a notion, of course, requires interaction, iterated temporally and spatially, with one another. As such, connectivity gives rise to globally emergent and interconnected phenomena. Social organizations are specific manifestations of connectivities that are always and already embedded within and across other various patterns of varying scales of organization, e.g., other families, 
communities or neighborhoods, municipalities and other settings of state. In other words, interactions alone, as temporal processes, shape on-going emerging patterns that give rise to varieties of patterns within and across many different scales of organizations. Such is the case in contexts of learning organizations like classrooms and schools.

\section{Healthy Collectivities}

Health is not a condition that one introspectively feels in oneself. Rather, it is a condition of being involved, of being in the world, of being together with one's fellow human beings, of active and rewarding engagement in one's everyday tasks. (Gadamer, 1996)

Wendell Berry (1995) has remarked that we must be seriously diseased for all of the talk that we hear about "health". Such a view may be cynical, naïve, narrow, unhelpful or even false, but it is hard to ignore, considering the nature of so many global problems. In fact, it is hard not to notice the many problems with the world and ourselves in times of great dis-ease. Whereas health, as its etymological root suggests, is concerned with notions like "healing" and "wholeness", disease and illness can, very much, make human beings conscious of the disconnectedness and isolation that come from a sense of unhealthiness (Ratson, 2003). In other words, health is not simply about biological bodies and how one feels in and with those bodies, it is ultimately about connectedness.

More traditional views of life, death, health and illness, however, are rooted in the everyday assumption of body-as-object that fills a particular space not shared by any other body. It follows, therefore, that our bodies, distinguishable from all other bodies, are thought of as containers. Thus, the origin and location of disease, as one might typically experience it, is rooted in the physical body, and is traditionally thought to be a malfunction of certain "building blocks" in the body which no longer work as they should. Conventional thought, therefore, suggests that illness is the result of some outside disturbance to one's inner structure. Of course, this sense of illness is not something that is accepted by everyone, although such expressions of illness are common experiences felt by many people.

This traditional view of health, as Dossey (1982) suggests, frames illness and disease as matters that can be treated in isolation from everything else. Moreover, as Ratson (2003, p. 15) remarks, "Modern medicine has advanced to the point where doctors can virtually ignore us and still do a pretty good job." One might conclude, therefore, that doctors as well need not invest much time to advance some kind of relationship with their patients. Disease and illness, thus, are things that happen to us as isolated and isolatable beings in the world at any time. Dossey continues: 
Bodies, as in the classical view of atoms, stand alone, both in space and in time. Although they form patterns, at heart they are single units in a deep, fundamental sense. Connectedness is seen only in terms of interaction of quintessentially separate bits and pieces. (p. 141)

This particular view of the world is proving to be rather limited and limiting. As the concern here is for the living, the notion that life, as a property of single bodies, does not fit well with the view that life is an emergent property of the entire universe, where all things are interconnected with one another. As such, there seems to be a certain measure of blindness, in a manner of speaking, of the greater connectivity in the world. Thus, in some sense, some healing is needed so that the usually invisible connections that hold us together bring forth a greater whole, as the notion of health suggests. In other words, at the heart of a view of healthy organizations of all scales is this notion of connections that all-at-once manifest various kinds of dynamical patterns. As Gregory Bateson (1979) writes, we are dealing with "patterns which connect."

Considering the adaptive nature of living phenomena, the notion of living organizations as learning organizations is not far away. Thus, it seems quite appropriate to consider, in a broad manner that addresses the nature of all kinds of living organizations, how and why the notion of connectivity might be an important principle for dynamical patterns of healthy organizations. What does a healthy learning organization, comprised as it is of (healthy) learners, look like?

\section{Learning and Healthy Social Organizations}

Many social organizations seem to be touting a shift toward or a greater emphasis on human relationships_-our ability to bring forth particular kinds of connections that serve the possibility for healthy learning organizations to emerge and unfold. The essence of such a move is perhaps more toward being a more cohesive organization. For instance, "closeness" speaks to a kind of intimacy as when one is physically/emotionally close to someone else. But sometimes people in intimate or meaningful relations "drift apart." This suggests that relationships may reflect different "strengths." Framed in this manner, "complicated" or "mechanical" organizations have weak connections or perhaps, quite simply, none at all. At the other end of the spectrum, a relation could be so strong that the possibility for action becomes rather limited, and a lock-step, rigid, predictable pattern emerges. It is, in fact, the relationships "in the middle" of these two extremes that are the kinds of relations that make for healthy organizations, where adaptation can happen and a kind of "dancing" between people is possible.

It is not so much that one should be concerned only with attempts to move toward the "middle" of these two extremes—at one end a "lock-step" 
relation and at the other end no relation at all—so that healthy relations in a healthy organization appear. Living systems inevitably manifest a wide range of different patterns-a reflection of their robustness and ability to "hang on." In certain human physiological patterns, for example, elderly human beings often have stable physiologies even though they may be frail. As Timothy Buchman (2002) writes, "It is not that aged patients have maladaptive responses to stress-rather their adaptive responses are inadequate." As we age, therefore, the connectedness that "breaks down" or weakens, gives rise to patterns that show either excessive order or uncorrelated randomness. In between the predictable stability of homeostatic processes and random fluctuations is a pattern of optimal connectedness which can be expressed in patterns of great variability. Moreover, such patterns of optimal connectedness are often noticed for particular forms: fractals (Gleick, 1988).

As such, one might recognize a healthy well-connected organization by attending to its form: is it fractal? But healthy organizations are not merely healthy because they are manifestations of particular forms. They are also connected to, and with, other healthy forms because living organizations need other living organizations to survive and sustain themselves. Thus, patterns of healthy organizations are the same patterns which give life to everything. But, even more, it is the pattern of all living things that are connected to one another in a massively entangled web of life.

\section{Intimations for the Health of Teachers and Education}

The implications for learning and healthy learning organizations, therefore, suggest a need to be attentive to the kinds of connections which appear simultaneously across many scales all-at-once. In the broad context of education, therefore, and specifically the project of schooling, the notions of healthy learning organizations and comparative dynamics open up the possibility for some compellingly different stances and perspectives for thinking about a number of different aspects of education and learning. These potentially include learning and its relation to the identity, practices and knowledge of learners; classroom dynamics; the framing and understanding of school subjects; curriculum design; pre-service programs for new educators; the influences of community and physical space; and, leadership to name some. Given that the concern here is for "connectivity", the remaining remarks in this paper will focus on the importance and relevance of the concept and notion of connectedness for education and, in particular, teachers.

Like the phrase, "No man is an island," no school, no classroom, no teacher ever stands alone. As Morrison (2002) remarks, "In schools, children are linked to families, teachers, peers, societies, and groups; teachers are linked to professional associations, other teachers, other providers of 
education, workplace placements for children, support agencies like psychological and social services, policy-making bodies, funding bodies, the courts and police services, and so on" (p. 18). In as much as a school may be seen as being and having a particular "body," it would seem clear that schools are relational patterns with connections within itself and with the world at large. And, in such a world, where there is often too much going on in terms of communication, say, the possibility for stress and teacher burnout is enormous, and the demands for a highly connected network create much chaos and ill-health.

Moreover, as Gabor Maté (2004, p. 34) writes, there are three factors that "universally lead to stress": uncertainty, the lack of information and the loss of control. These three factors, in fact, are present in the lives of all people with chronic stress. Moreover, these three factors speak to an "absence that the [teacher] perceives as necessary for survival" (p. 34). Put differently, there is a loss of connectedness. Teachers-especially new and pre-service teachers-speak of these matters in a myriad of different ways.

For myself, I have not only experienced these matters first hand, but as a teacher-educator in a pre-service teacher education program, I have seen this in the program's teacher candidates. It is quite hard to miss. After all, as human beings, we do tend to notice occasions of dis-ease rather than health. There is, for some, a rather pervasive idea that matters related to teaching and learning should rest upon the ideas of certainty, clarity and absolute control. These are, in fact, the exact opposite of what Maté talks about in terms of stress and burnout. This swing of the proverbial pendulum, however, does not make things any better. In fact, there is a need for some kind of "middle ground" where teachers and all involved might find greater health. Thus, some measure of connectivity is required, but not too little nor too much.

In addition, while a certain degree of connectivity is required for healthy self-organizing structures, like a teacher, classroom or school, the connectivity of any healthy organization must reflect a "distributed knowledge system" (Morrison, 2002, p. 18). In other words, the location and control of information, knowledge, and meaning must not be centrally located in a command-and-control environment, but distributed, shared and circulated through the organization itself. To be sure, as if it were possible, no teacher, principal, student, parent or ministerial body can be the holder of all knowledge. In fact, some measure of democracy must be present where all can and must participate in the co-creation of the larger organization. And, moreover, self-organization must be a part of that process. If one does not feel in control, then one is most likely being controlled. Put differently, there is a lack of autonomy and, yet, paradoxically speaking, all people need to be connected in some way, although autonomy does not simply imply disconnectedness and being able to do whatever one wants. 
Of course, one should not think of leadership as gate-keeping, directing, or preserving the senior figure of a school, but rather as something which is distributed, shared and circulated. But such matters require flexibility and adaptability for survival. They also require feedback within, and iteration throughout, the organization to function well enough. Certainly, there are many other principles at work in an organization that play a part in its health. For now, however, the importance of connectivity, expressed in particular ways, cannot be overlooked as it is a vital principle of, and for, living organizations. Whether there is too little or too much connectivity, dis-ease and / or toxic relations are bound to be present. But where there is just enough, we should find a robust organization living on the edge of chaos.

\section{Acknowledgments}

A version of this paper was presented at the $3^{\text {rd }}$ Annual Complexity Science and Educational Research (CSER) conference in Robert, Louisiana on November 20-22, 2005.

I also wish to express my thanks to the anonymous reviewers of this paper and my Associate Dean for their comments and suggestions which have served to clarify a number of points and aspects of this paper.

\section{References}

Aubin, David, \& Amy Dahan Dalmedico. 2002. Writing the history of dynamical systems and chaos: Longue Durée and revolution, disciplines and cultures. Historia Mathematica 29: 273-339.

Bateson, Gregory. 1979. Mind and nature: A necessary unity. New York: E.P. Dutton.

Berry, Wendell. 1995. Health is membership. In Another turn of the crank: Essays. Washington, DC: Counterpoint.

Buchman, Timothy G. 2002. The community of self. Nature 420(14): 246-251.

Dossey, Larry. 1982. Space, time, and medicine. Boston, MA: Shambhala.

Frost, Peter J. 2003. Toxic emotions at work: How compassionate managers handle pain and conflict. Boston: Harvard Business School Press.

Gadamer, Hans-Georg. 1996. The enigma of health: The art of healing in a scientific age. Translated by Jason Gaiger \& N. Walker. Stanford, CA: Stanford University Press.

Gleick, James. 1988. Chaos: Making a new science. New York: Penguin.

Goldberger, Ary L., Luis A. N. Amaaral, Jeffrey M. Hausdorff, Plamen Ch. Ivanov, and C. -K. Peng. 2002. Fractal dynamics in physiology: Alterations with disease and aging. Proceedings of the National Academy of Sciences (Online) 99 (1 (Suppl.)): 2466-2472.

Hausdorff, Jeffrey M., Susan L. Mitchell, Renee Firtion, C. -K. Peng, Merit E. Cudkowicz, Jeanne Y. Wei, \& Ary L. Goldberger. 1997. Altered fractal dynamics of gait: Reduced stride-interval correlations with aging and Huntington's Disease. Journal of Applied Physiology 82: 262-269.

Kauffman, Stuart A. 1995. At home in the universe: The search for laws of self-organization and complexity. New York: Oxford University Press.

Kelso, J. A. Scott, \& David A. Engstrøm. 2006. The complementary nature. Cambridge, 
MA: The MIT Press.

Maté, Gabor. 2004. When the body says no: The cost of hidden stress. Toronto: Vintage.

Morrison, Keith. 2002. School leadership and complexity theory. London: Routledge Falmer.

Ratson, Gary A. 2003. The meaning of health: The experience of a lifetime. Victoria, BC: Trafford.

Stacey, Ralph D. 2003. Complexity and group processes: A radically social understanding of individuals. New York: Brunner-Routledge.

Stanley, R. Darren. 2004. The body of a 'healthy' education system. Journal of Curriculum Theorizing 20(4): 63-74.

Stanley, R. Darren. 2005. Toward a view of healthy learning organizations through complexity. Unpublished doctoral dissertation. Edmonton, AB: University of Alberta.

\section{About the Author}

Darren Stanley is Assistant Professor of Elementary Education (Mathematics) in the Faculty of Education at the University of Windsor in Windsor, Ontario. He received his doctorate form the University of Alberta, and his research interests include the study of complex phenomena as a paradigm for understanding and framing aspects of health and healthy learning organizations, including healthy schools. Additionally, he is interested in the circulation of lived phenomena with complexity frameworks. As a teacher educator in the faculty's pre-service education program, he is interested in the ways in which pre-service teachers enact complexified understandings of mathematics and pedagogy. (E-mail address: dstanley@uwindsor.ca)

(C) Copyright 2006. The author, DARREN STANLEY, assigns to the University of Alberta and other educational and non-profit institutions a non-exclusive license to use this document for personal use and in courses of instruction provided that the article is used in full and this copyright statement is reproduced. The authors also grant a non-exclusive license to the University of Alberta to publish this document in full on the World Wide Web, and for the document to be published on mirrors on the World Wide Web. Any other usage is prohibited without the express permission of the authors. 